\title{
Essais
}

Revue interdisciplinaire d'Humanités

$6 \mid 2015$

L'histoire par les lieux

\section{Giuliano Milani, Bologna}

\section{Marco Conti}

\section{(2) OpenEdition}

Journals

Édition électronique

URL : http://journals.openedition.org/essais/6057

DOI : 10.4000/essais.6057

ISSN : 2276-0970

\section{Éditeur}

École doctorale Montaigne Humanités

\section{Édition imprimée}

Date de publication : 15 janvier 2015

Pagination : 194-196

ISBN : 978-2-9544269-4-5

ISSN : 2417-4211

\section{Référence électronique}

Marco Conti, «Giuliano Milani, Bologna », Essais [En ligne], 6 | 2015, mis en ligne le 30 novembre 2020, consulté le 10 décembre 2020. URL : http://journals.openedition.org/essais/6057 ; DOI : https:// doi.org/10.4000/essais.6057 


\section{Comptes rendus}

Giuliano Milani, Bologna, Spoleto, Fondazione Cisam, 2012.

Dans la plupart des villes italiennes, le Moyen-Âge a laissé une trace importante tant dans les documents d'Archive que dans le paysage urbain. Depuis 2009, la fondation CISAM (Centro italiano di Studi sull'alto Medioevo) a créé une collection intitulée "Il Medioevo nelle città italiane ${ }^{1}$. Ces publications offrent principalement aux étudiants et aux jeunes chercheurs une première approche d'une série de villes italiennes, avec une vision du paysage urbain et des œuvres d'art, accompagnée d'un choix de sources écrites relatif au Moyen-Âge. Un des derniers livres à être publié dans cette collection est celui de Giuliano Milani sur Bologne. Giuliano Milani est chercheur en Histoire Médiévale à l'Università La Sapienza di Roma. Il s'intéresse notamment aux pratiques institutionnelles des communes italiennes entre le XII ${ }^{e}$ et $\mathrm{XIV}^{e}$ siècle, à l'exclusion des ennemis politiques, aux mécanismes de gouvernement, à la justice et à l'utilisation pratique de l'écriture et des images ${ }^{2}$.

L'Introduction de cet ouvrage renvoie l'image que tout le monde voit en arrivant pour la première fois à Bologne, c'est à dire, celle d'un centre-ville resté entièrement médiéval. En regardant les monuments et les lieux principaux de Bologne : Piazza Maggiore, San Petronio, le Palazzo del re Enzo, le Palazzo della Mercanzia, la tour des Asinelli, etc., l'aspect moyenâgeux de la ville saute aux yeux, mais il est mensonger. $\mathrm{Si}$, d'un côté, cet aspect est le résultat d'une continuité entre cette époque et nos jours, de l'autre il résulte de choix dont le but a été la célébration de son passé. Ce processus s'est produit à différents moments : comme à la fin du Moyen-Âge avec la sélection des

1 Actuellement ils ont été publiés sur la ville de Sienne, Fermo, Bologne, Ascoli Piceno, Trento et Gênes.

2 G. Milani, I comuni italiani, Roma-Bari, Laterza, 2005 ; et L'esclusione dal comune. Conflitti e bandi politici a Bologna e in altre città italiane tra XII XIVE secolo, Roma, ISIME, 2003. 
bâtiments à démolir ou des nouveaux édifices à bâtir ; ou à la Belle Époque avec les restaurations (et les modifications) de l'architecte Alfonso Rubbiani, et la démolition d'une partie des tours médiévales.

Le premier chapitre se propose de tracer le profil général de la ville. L'auteur nous guide dans la ville dans le but de nous montrer l'expansion et les changements de Bologne depuis l'époque romaine, à travers une brève étude de l'évolution du territoire et de la démographie de la ville. Une place importante est dédiée à la reconstruction de son profil historique. Les informations relatives à l'époque romane et au haut Moyen-Âge, nous présentent une ville affligée par de nombreuses guerres, mais qui, comme le montrent les données archéologiques, a su conserver une vie économique et une société urbaine.

Pendant la conquête des Lombards, et la domination des Carolingiens, le territoire de la ville résulte fortement fragmenté du point de vue juridictionnel, en raison de l'existence d'une compétition politique et économique entre d'un côté les pouvoirs extérieurs et d'un autre côté les pouvoirs locaux qui commençaient la construction d'un grand patrimoine.

Ces pouvoirs se renforcèrent pendant la période de la querelle des Investitures, donnant lieu à un jeu politique très articulé dans lequel se trouvent : des nobles de tradition civique, le roi d'Italie, les puissant évêques de Bologne et des villes environnantes. Pendant cette période Bologne suivra un parcours politique similaire aux autres villes de la plaine du Pô : du côté de l'empereur dans un premier temps, ensuite, après l'imposition de podestats impériaux, la rébellion à l'empire, l'adhésion à la Ligue Lombarde jusqu'à la paix de Constance. Ensuite, il est analysé toute l'évolution de la période communale, avec ses épisodiques dominations étrangères et de crypto-seigneuries (Pepoli, Visconti, légats apostoliques, etc.), jusqu’à la seigneurie des Bentivoglio.

Le second chapitre, consacré au très riche patrimoine documentaire de la ville, est divisé en quatre parties. La première donne des informations sur des lieux actuels de conservation de cette documentation (Archivio di Stato di Bologna, Biblioteca comunale dell'Archiginasio, etc.). Dans la deuxième, l'auteur analyse d'abord les narrations hagiographiques et les sources historiographiques (aussi bien les chroniques du XIII"e et XIV ${ }^{e}$ siècles que les écrits de la première époque moderne, essentiels à la reconstruction de la Bologne médiévale). L'auteur illustre ensuite les sources disponibles sur l'Épiscopat, le Chapitre de la Cathédrale, d'autres centres religieux de la ville, d'une grande richesse eu égard au rapport complexe que la ville entretient avec la Papauté. La troisième partie examine les sources de l'époque de la commune, avec une présentation des pratiques documentaires relatives à chaque période clé de son évolution. La quatrième partie est dédiée à la documentation relative aux corporations, aux guildes, aux hôpitaux, à l'université, et aux autres associations qui ont joué un rôle de premier plan dans l'histoire de Bologne. Les derniers paragraphes traitent brièvement des fonds notariaux, des fonds privés importants et des sources miscellanées. 
Le troisième chapitre est consacré au paysage urbain et aux œuvres d'art de la ville. L'auteur nous décrit les monuments principaux de la ville en rapport à chaque période de l'époque médiévale. Bologne, grâce à sa puissance économique entre $\mathrm{XIII}^{\mathrm{e}}$ et $\mathrm{XIV}^{\mathrm{e}}$ siècles, a pu planifier une importante stratégie de construction et de décoration de la ville. Le Moyen-Âge matériel et artistique de Bologne est délimité entre deux discontinuités : la première dans les dernières décennies du $\mathrm{III}^{\mathrm{e}}$ siècle et la moitié du $\mathrm{IV}^{\mathrm{e}}$ siècle, coïncide avec la fin de la ville romaine ; la seconde, à la moitié du XVe siècle, avec l'introduction de nouveaux styles et matériaux qui restent bien ancrés dans la tradition des siècles précédents. Bologne a une hérédité médiévale compliquée, et son paysage monumental est absolument hégémonique depuis le $\mathrm{XIII}^{\mathrm{e}}$ au $\mathrm{XV}^{\mathrm{e}}$ siècles, même si, comme nous l'avons vu, il a été falsifié par la suite.

Cet ouvrage est un bon outil de recherche, qui avec simplicité mais jamais avec superficialité, nous présente la ville de Bologne et son histoire. Un parcours et un guide, très utile pour nous orienter dans l'abondante documentation conservée, relative à l'époque médiévale, complétée par une bibliographie mise à jour. Une très belle perle, en somme, qui va s'ajouter à la collana il "Medioevo nelle città italiane ".

\section{Marco Conti}

ED 483 ScSo (CIHAM)

Université Lumière Lyon 2

Marco.Conti@univ-lyon2.fr

Daniela Visone, La Nascita del nuovo teatro in Italia 1959-1967, Corazzano (Pise), Titivillus, 2010, p. 328.

« Nouveau Théâtre » est une définition relativement récente en Italie, qui, à coté du " théâtre d'avant-garde " et du " théâtre expérimental ", porte un regard autre sur les expériences théâtrales " différentes ", " excentriques ", nées pendant les années soixante.

Dans ce cadre, le projet dirigé par Lorenzo Mango, professeur à l'Université de Naples "L'Orientale », est une contribution du plus grand intérêt pour l'histoire des études théâtrales. Daniela Visione en a fourni le premier volet, avec la publication de sa thèse en 2010 : Il Nuovo Teatro in Italia 19591967. Salvatore Margiotta a récemment publié le deuxième volet (Il Nuovo Teatro in Italia 1967-1975) $)^{3}$ et on attend la publication des recherches de Mimma Valentino (Il Nuovo Teatro in Itala 1975-1989).

3 Salvatore Margiotta, Il Nuovo Teatro in Italia 1968-1975, Corazzano, Titivillus, 2013, p. 480. 